\title{
Transient receptor potential (TRP) gene superfamily encoding cation channels
}

\author{
Zan Pan, ${ }^{*}$ Hua Yang ${ }^{2}$ and Peter S. Reinach ${ }^{2}$ \\ 'Margaret Dyson Vision Institute, Weill Cornell Medical College, 1300 York Avenue, New York, NY 10065, USA \\ ${ }^{2}$ Department of Biological Science, State University of New York, College of Optometry, 33 West 42nd Street, New York, \\ NY 10036, USA \\ *Correspondence to: Tel: +1 212746 2382; Fax: +1 212746 811; E-mail: zap2001@med.cornell.edu
}

Date received (in revised form): 8th December 2010

\begin{abstract}
Transient receptor potential (TRP) non-selective cation channels constitute a superfamily, which contains 28 different genes. In mammals, this superfamily is divided into six subfamilies based on differences in amino acid sequence homology between the different gene products. Proteins within a subfamily aggregate to form heteromeric or homomeric tetrameric configurations. These different groupings have very variable permeability ratios for calcium versus sodium ions. TRP expression is widely distributed in neuronal tissues, as well as a host of other tissues, including epithelial and endothelial cells. They are activated by environmental stresses that include tissue injury, changes in temperature, $\mathrm{pH}$ and osmolarity, as well as volatile chemicals, cytokines and plant compounds. Their activation induces, via intracellular calcium signalling, a host of responses, including stimulation of cell proliferation, migration, regulatory volume behaviour and the release of a host of cytokines. Their activation is greatly potentiated by phospholipase C (PLC) activation mediated by coupled GTP-binding proteins and tyrosine receptors. In addition to their importance in maintaining tissue homeostasis, some of these responses may involve various underlying diseases. Given the wealth of literature describing the multiple roles of TRP in physiology in a very wide range of different mammalian tissues, this review limits itself to the literature describing the multiple roles of TRP channels in different ocular tissues. Accordingly, their importance to the corneal, trabecular meshwork, lens, ciliary muscle, retinal, microglial and retinal pigment epithelial physiology and pathology is reviewed.
\end{abstract}

Keywords: TRP, cornea, glaucoma, cataract, photoreceptor, retina, eye

\section{Introduction}

The founding member of the transient receptor potential (TRP) protein superfamily was first described in Drosophila. In some of these flies, prolonged exposure to light only induced transient photoreceptor depolarisation, whereas in the wild type the response was sustained. This difference was attributed to mutant trp gene expression. Mammalian TRP homologues have been identified over the past 30 years since the initial trp description in Drosophila. The members of this superfamily constitute a large and very different collection of proteins that are expressed in many tissues and cell types. ${ }^{1}$ This superfamily is conserved throughout evolution, from nematodes to humans. ${ }^{2}$ They form non-selective monovalent and divalent cation channels with very variable $\mathrm{Ca}^{2+} / \mathrm{Na}^{+}$permeability ratios. Some members are even impermeable for $\mathrm{Ca}^{2+}$, whereas others are highly $\mathrm{Ca}^{2+}$ permeable relative to monovalent cations. TRPV5 and -6 exhibit a $\mathrm{Ca}^{2+} / \mathrm{Na}^{+}$permeability ratio of greater than $100 .^{3}$ This variability is unique by comparison with most other ion channel families. In those 
cases, the differences in permeation properties within a single family are for the most part very much smaller. ${ }^{4}$ TRPs are distinguished from one another based on differences in their primary amino acid sequence rather than ligand affinity or selectivity. This system of classification is used because their properties are heterogeneous and their regulation is complex. The common feature of TRP channels is that they comprise six putative transmembrane spanning domains and a cationpermeable pore formed by a short hydrophobic region between transmembrane domains 5 and 6. They assemble themselves as homo-or heterotetramers to form cation channels (Figure 1). These channels are activated by a remarkable assemblage of very diverse stimuli.

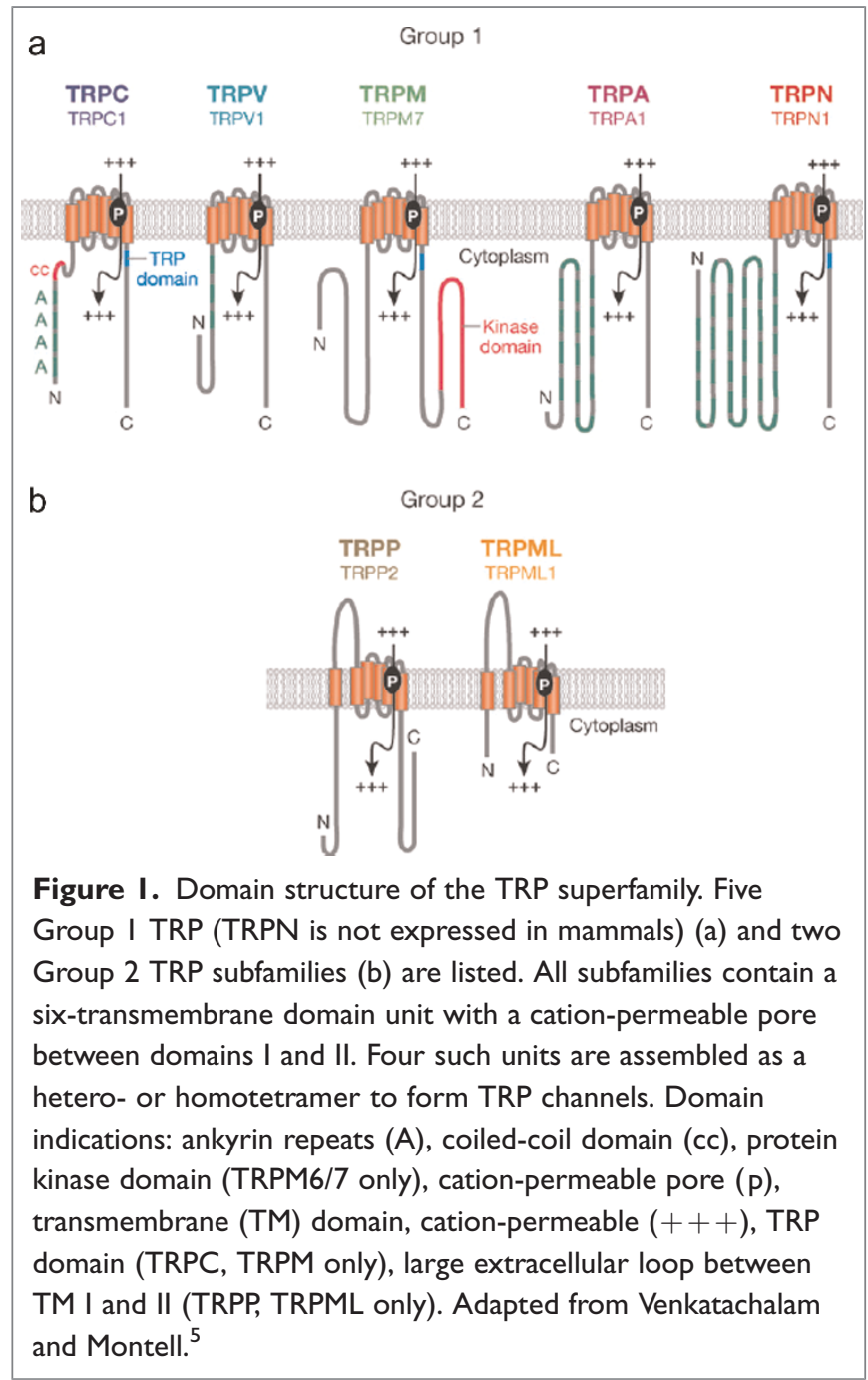

Genetic ablation studies in worms, flies and mice indicate that TRPs serve as sensors to elicit responses to a variety of stimuli, ranging from temperature, osmotic pressure, olfaction, taste, mechanical stress, light and injury-induced inflammation mediating pain. These channels are also activated by intracellular and extracellular messengers, as well as declines in the calcium content of intracellular $\mathrm{Ca}^{2+}$ stores. $^{6}$ The physiological importance of TRP expression is indicated by the finding that TRP mutations are linked to human diseases. ${ }^{7}$

In humans, 27 different TRP genes are grouped into six different subfamilies based on their amino acid homologies (Table 1). The nomenclature of the TRP superfamily has been evolving for many years. Many genes and proteins within this superfamily have been referred to by multiple names, and in some cases two different genes belonging to different subfamilies have shared the same name. To overcome such confusion, a unified TRP superfamily nomenclature system was published in 2003, based on the TRPC ('Canonical'), TRPM ('Melastatin'), TRPV ('Vanilloid') subfamilies, with TRPA ('Ankyrin') being assigned subsequently. ${ }^{8}$ These TRP subfamilies are designated as Group 1 TRPs. The nomenclature of the genes of Group 2 TRPs is based on the human phenotypes that the founding genes cause when mutated polycystic kinase disease (PKD) and mucolipidosis type IV (MCOLN, Mucolipin) — while their encoded proteins have also been referred to as TRPP ('Polycystin') and TRPML ('Mucolipin'), respectively. Although the Group 2 proteins appear to fall into the same superfamily as Group 1 TRPs, Group 2 and Group 1 genes are only distally related (Figure 2).

Group 1 TRPs share substantial sequence homology in the transmembrane domains. The TRPC subfamily consists of seven genes (ie TRPC1-7). TRPC2 is a pseudogene in humans. The TRPM subfamily comprises eight genes (ie TRPM 1-8), of which three encode channel-like proteins and five non-channel proteins. The TRPV subfamily contains six members (ie TRPV1-6). Recently, the TRPA subfamily was delineated and has one member. ${ }^{9}$ TRPC, TRPV and TRPA channels 
Table I. Human TRP genes. Adapted from Birnbaumer et al. ${ }^{10}$

\begin{tabular}{|c|c|c|c|c|c|c|}
\hline $\begin{array}{l}\text { TRP } \\
\text { Gene }\end{array}$ & $\begin{array}{l}\text { TRP } \\
\text { Protein }\end{array}$ & $\begin{array}{l}\text { Genome } \\
\text { Accession } \\
\text { Number }\end{array}$ & $\begin{array}{l}\text { mRNA } \\
\text { Accession } \\
\text { Number }\end{array}$ & $\begin{array}{l}\text { Gene Locus in } \\
\text { Contig/Gene }\end{array}$ & Locus & $\begin{array}{l}\text { \# of } \\
\text { Exons }\end{array}$ \\
\hline TRPCI & TRPCI & NT_005832 & U31II0 & $3300676-3384487$ & $3 q 22-q 24$ & 13 \\
\hline TRPC3 & TRPC3 & NT_016354 & U47050 & C II488244-II542672 & $4 q 27$ & 11 \\
\hline TRPC4 & TRPC4 & NT_033922 & AFI75406 & C 6785I34-70I8278 & $13 q|3.1-q| 3.2$ & 13 \\
\hline TRPC5 & TRPC5 & NT_025319 & AF054568 & C 2989I79-329764I & $\mathrm{Xq} 23$ & 10 \\
\hline TRPC6 & TRPC6 & NT_009|5I & AF080394 & C $4866094-4998380$ & $1|q 2|-q 22$ & 13 \\
\hline TRPC7 & TRPC7 & NT_037664 & AJ272034 & C 424567-56864I & $5 q 31.1$ & 11 \\
\hline TRPMI & TRPMI & NT_010363 & AF07I 787 & C 2085I $78-2 \mid 85564$ & $15 q|3-q| 4$ & 27 \\
\hline TRPM2 & TRPM2 & NT_0II5I5 & $\mathrm{AB} 001535$ & $1085877-\mid 175698$ & $21 \mathrm{q} 22.3$ & 32 \\
\hline TRPM3 & TRPM3 & NT_008580 & AJ505025 & C 23।4499-2399547 & $9 q 21.11$ & II \\
\hline TRPM4 & TRPM4 & NT_0III09 & AF497623 & | | $792968-|| 847007$ & $19 q 13.32$ & 29 \\
\hline TRPM5 & TRPM5 & NT_033238 & AFI77473 & C 573099-591648 & IIpI5.5 & 24 \\
\hline TRPM6 & TRPM6 & NT_008580 & AF448232 & C $6501941-6667540$ & $9 q 21.13$ & 39 \\
\hline TRPM7 & TRPM7 & NT_010194 & AF346629 & C 5824294-5950796 & $15 q 21$ & 39 \\
\hline TRPM8 & TRPM8 & NT_005I 20 & AY090109 & $7565 \mid 4-858635$ & $2 q 37.1$ & 25 \\
\hline TRPVI & TRPVI & NT_010692 & AYI3I 289 & C $2321912-2365875$ & $17 p \mid 3.3$ & 17 \\
\hline TRPV2 & TRPV2 & NT_010718 & AJ487963 & $9021374-9042801$ & $|7 p| \mid .2$ & 15 \\
\hline TRPV3 & TRPV3 & NT_010692 & AYII 8268 & C $2269657-23$ I 4460 & 17p|3.3 & 18 \\
\hline TRPV4 & TRPV4 & NT_009770 & A)296305 & C 790403-84072I & $12 q 23-q 24.1$ & 15 \\
\hline TRPV5 & TRPV5 & NT_0079|4 & AJ27/ 207 & C $3218677-3244315$ & $7 q 35$ & 15 \\
\hline TRPV6 & TRPV6 & NT_0079|4 & AF365927 & C $3182370-3196917$ & $7 q 33-q 34$ & 15 \\
\hline TRPAI & TRPAI & NT_008183.18 & YI060I & C2484I572-24786439 & $8 q 13$ & 27 \\
\hline PKD2 & TRPP2 & NT_006204 & U50928 & $764375|-77| 3856$ & $4 q 21-23$ & 15 \\
\hline PKD2LI & TRPP3 & NT_030059 & AF07348I & C 73I2540-7354872 & $10 q 24$ & 16 \\
\hline PKD2L2 & TRPP5 & NT_0167|4 & AFII 8125 & $365617-420393$ & $5 q 31$ & 14 \\
\hline MCOLNI & TRPMLI & NT_0778I2.2 & AF287269 & $191108-203260$ & $19 p|3.3-p| 3.2$ & 13 \\
\hline MCOLN2 & TRPML2 & NT_032977.7 & AY083533 & $39282589-39210261$ & Ip22 & 13 \\
\hline MCOLN3 & TRPML3 & NC_00000I & AF475085 & C85226190-85195787 & Ip22.3 & 13 \\
\hline
\end{tabular}

*TRPPI accession number used for multiple sequence alignment analysis.

contain ankyrin repeats in the intracellular domain' in the region of the $\mathrm{C}$ terminal near the N-terminal domain, whereas the TRPC and putative transmembrane segment. TRPM6 and -7 TRPM subfamilies possess a proline-rich 'TRP have a protein kinase domain in the $\mathrm{C}$ terminal. 


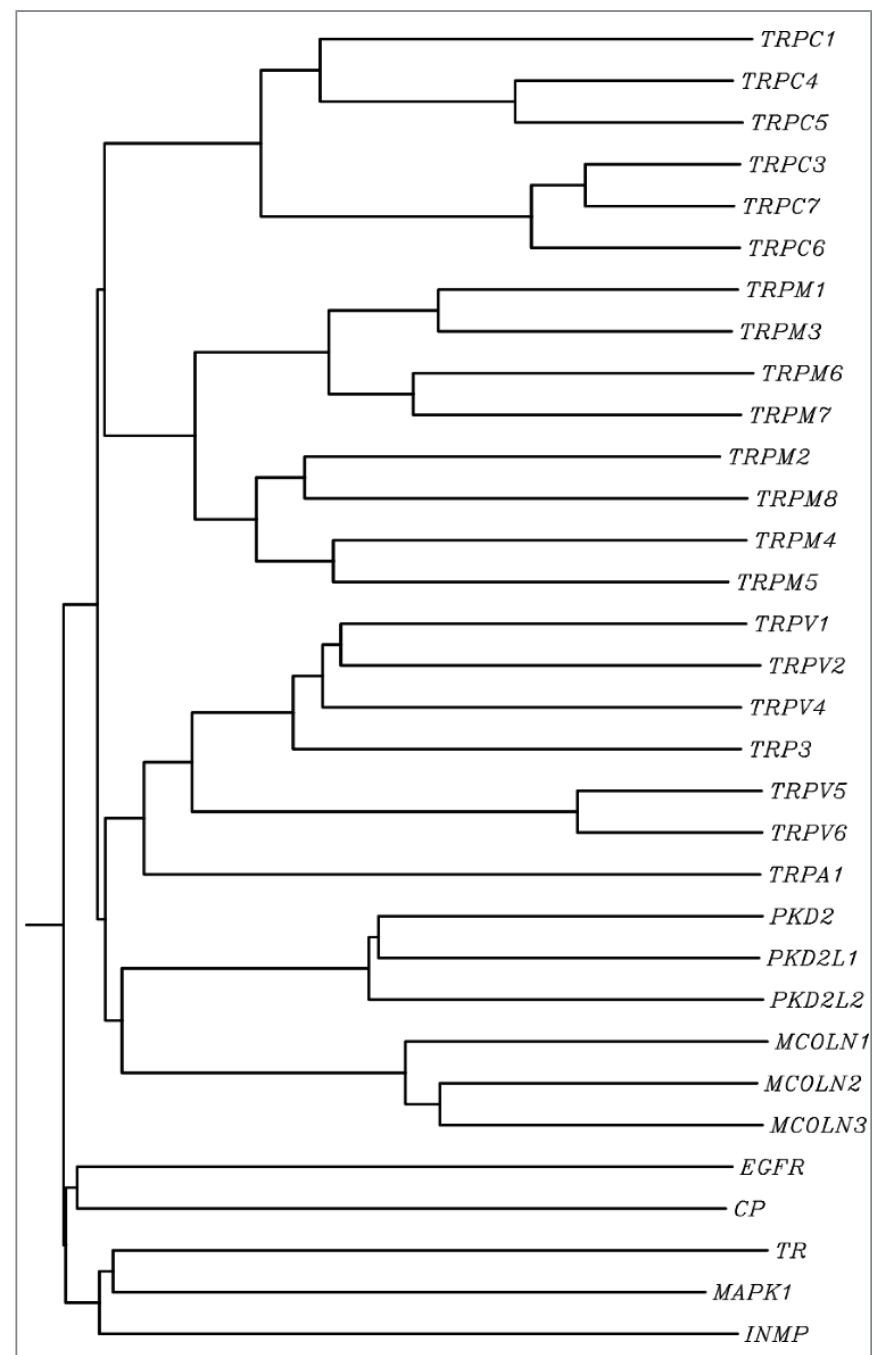

Figure 2. The dendrogram of the TRP superfamily in humans. Random proteins bound to the plasma membrane (ie EGF receptor, EGFR), endoplasmic reticulum (ie calreticulin precursor, $\mathrm{CP}$ ), mitochondria (thioredoxin reductase, TR), nuclear membrane (ie inner nuclear membrane protein, INMP) and a cystolic protein (ie mitogen-activated protein kinase I, MAPKI) were selected to determine if PKD and MCOLN are evolutionarily related to TRP or the results of convergent evolution. Note that PKD and MCOLN belong to TRP superfamilies, since random genes extend from branches distinct from the TRP superfamily.

The PKD and MCOLN gene branches encoding TRPP and TRPML channel proteins, respectively, belong to Group 2 TRPs. They are distally related to Group 1, as they contain limited sequence homology to Group 1 TRPs, although such homology is still greater than that exhibited by random genes with Group 1 TRPs (Figure 2). TRPP proteins share 25 per cent amino acid sequence similarity to TRPC3 and TRPC6 over a region including transmembrane domains 4 and 5 and the hydrophobic pore loop between domains 5 and 6 . The three TRPML proteins are small compared to other proteins. Homology of their amino acid sequence with TRPC proteins is limited to the region spanning transmembrane domains 4 to 6 (amino acids 331 to 521). Group 2 TRPs have a unique large extracellular loop between their first and second transmembrane domains. Nevertheless, they are still named as TRPs, because they have six transmembrane domains and are permeable to cations.

The emerging realisation of the broad spectrum of responses that are elicited by TRP activation has prompted extensive research into identifying novel strategies to manipulate their activation in various disease states. This review focuses on the outcome of numerous studies that have shown that modulation of TRP function may provide a novel approach for treating some ocular diseases.

\section{Cornea}

Corneal transparency is dependent on continuous renewal of its outermost epithelial layer. This process is necessary to maintain epithelial integrity and a smooth optical surface. These attributes assure preservation of visual acuity and enable the epithelial layer to provide a barrier function, which protects the underlying stroma from pathogenic invasion. This protective function can be compromised by dysregulated inflammation occurring in anterior ocular surface diseases. Such an outcome can lead to the development of corneal opacity, severe pain, perforation and neovascularisation. Any of these outcomes may require corneal transplantation surgery if therapeutic agents cannot reverse these inappropriate responses to infection or injury. Currently, the treatment of corneal disturbances is limited to the use of steroids, which can have adverse effects.

The cornea has the highest sensory innervation density of any tissue in the body. Until recently, the nociceptive and thermal sensory functions were thought to be limited to corneal afferent sensory 
nerves. ${ }^{11}$ As no functional evaluation was performed, it was suggested that TRPV1 only participates in eliciting healing and nociceptive transduction. More recent studies directly demonstrate that there is functional expression of TRPV1-4, as well as TRPC4, in the epithelial layer. ${ }^{12-17}$ In the epithelium, TRPV1 activation by capsaicin, a selective agonist, induces responses that support hastening of corneal epithelial wound healing following injury, as well as inducing inflammation through increases in the expression of interleukin (IL)-6 and IL-8, which are proinflammatory and chemoattractant cytokines. ${ }^{12}$ Over the short term, inflammation and infiltration have adaptive value, since they serve to suppress pathogenic invasion. Concurrently, TRPV1 induces increases in epithelial cell proliferation and migration, both of which hasten restoration of the epithelial barrier function. TRPV1-4 isoforms also serve as thermosensors over defined temperature ranges. In addition, TRPV3 activation, either by temperatures above $33^{\circ} \mathrm{C}$ or exposure to the selective agonist, carvacrol, induces currents that are, in part, accounted for by increases in $\mathrm{Ca}^{2+}$ influx. ${ }^{13,14}$ TRPV4 serves as an osmosensor to mediate a regulatory volume decrease (RVD) response to a hypotonic challenge, since knockdown of its expression in human corneal epithelial cells (HCECs) blunts cell volume restoration. ${ }^{15}$ A hallmark of dry eye disease is that the anterior ocular surface is exposed to hypertonic tears. Such stress is frequently associated with chronic inflammation, resulting, in part, from hypertonic-induced increases in IL-6 and IL-8 release. To determine if TRPV1 could mediate such increases, HCECs were exposed to the same range of osmolarities measured in the tears of patients afflicted with dry eye disease. TRPV1 activation contributes to the increases in IL-6 and IL-8 release through nuclear factor $\kappa \mathrm{B}$ $(\mathrm{NF}-\kappa \mathrm{B})$ activation, since, during exposure to a TRPV1 antagonist, these increases are fully suppressed. $^{16}$ These results suggest that blocking TRPV1 activation during chronic exposure to hypertonic tears in dry eye disease may have therapeutic value in reducing inflammation. TRPV1 stimulation also induces increases in HCEC proliferation, migration and IL-6 and IL-8 release through transactivation of the epithelial growth factor (EGF) receptor. ${ }^{16,17}$ TRPC4 activation by $\mathrm{EGF}$ is required for this mitogen to stimulate HCEC proliferation, since knockdown of TRPC4 expression blunts a proliferative response. ${ }^{18}$ Such a reduction is expected to delay restoration of corneal epithelial integrity. In addition, there is functional expression TRPC4 and TRPV1-3 in the single-celled endothelial layer. ${ }^{19,20}$ Taken together, these studies in cultured corneal epithelial and endothelial cells provide additional proof that TRP channels are crucial components for maintaining corneal transparency and eliciting adaptive responses to stress. This realisation makes TRP channels important targets for the treatment of diseases arising from their malfunction and for better management of inflammatory pain.

\section{Trabecular meshwork}

The trabecular meshwork (TM) is located in the angle formed by the cornea and the iris. The TM contains contractile elements that control fluid drainage from the anterior chamber into the canal of Schlemm. In addition, the tension on the meshwork is also under the control of the tension exerted by the ciliary muscle. Malfunction of meshwork contractility often leads to ocular hypertension and glaucoma. ${ }^{21}$ The TM cells have surface receptors that are activated by substances released by TM cells and surrounding tissues. Substances that activate these receptors induce contraction by stimulating $\mathrm{Ca}^{2+}$ influx through different transplasma membrane calcium pathways. An increase in the TM contractile state as a result of $\mathrm{Ca}^{2+}$ transient increases the resistance to aqueous humour outflow. In TM cells, there are two types of store-operated calcium entry (SOCE) pathways that are activated by either bradykinin (BK) or endothelin-1 (ET-1) stimulation of their cognate receptors. $^{22}$ The activity of SOCE pathways is inversely related to the $\mathrm{Ca}^{2+}$ filling state of intracellular stores (ICS). SOCE activation occurs following receptor-induced increases in inositol 1,4,5-trisphosphate $\left(\mathrm{IP}_{3}\right)$ levels resulting from 
phospholipase C (PLC)-induced phosphatidyl inositol 4,5-bisphosphate $\left(\mathrm{PIP}_{2}\right)$ hydrolysis. $\mathrm{IP}_{3}$, in turn, interacts with its cognate receptor on ICS membranes, leading to depletion of ICS $\mathrm{Ca}^{2+}$ content. Communication between the store-operated $\mathrm{Ca}^{2+}$ channel activity and the $\mathrm{Ca}^{2+}$ level of ICS is mediated through stromal interaction molecular 1 (STIM1) interaction with Orai 1. Orai 1 is a pore subunit SOCE, located in the plasma membrane. Upon a decline in the ICS $\mathrm{Ca}^{2+}$ content, STIM1 migrates to interact with Orai 1, which is required for SOCE activation. One of the SOCEs involves the calcium release-activated calcium channel and another involves TRPC-containing channels. The two types of TRPCs implicated are TRPC1 and TRPC4. These two different types of $\mathrm{Ca}^{2+}$ pathways may contribute to modulating cell contractility or to releasing different substances that regulate outflow facility as a local homeostatic mechanism.

\section{Ciliary muscle}

Changes in the ciliary muscle contractile state are essential for eliciting lens accommodation and regulation of aqueous humour outflow. This muscle is densely innervated by parasympathetic neurones which, via acetylcholine release, induce muscarinic receptor stimulation on the surface of the muscle cell membrane. The contractile state of the ciliary muscle has a direct effect on the volume of fluid exiting from the anterior chamber through the conventional outflow route. Such outflow is regulated through a functional antagonism between the ciliary muscle and the TM. Relaxation of the ciliary muscle leads to lens accommodation, whereas contraction induces TM distension with a subsequent reduction in aqueous humour outflow. $^{23}$ Ciliary muscle contraction induced by acetylcholine is, in turn, elicited by sustained $\mathrm{Ca}^{2+}$ influx through the cell membrane. There are at least two different types of $\mathrm{Ca}^{2+}$ influx pathways that can be activated by muscarinic receptor stimulation. One possibility is that influx occurs through receptor-operated channels. $\mathrm{Ca}^{2+}$ influx through this pathway is dependent on receptor occupation by agonists. Another alternative is SOCE. ${ }^{24}$ In bovine ciliary muscle, gene expression and immunolocalisation analysis revealed the presence of TRPC1, TRPC3, TRPC4 and TRPC6. These TRPs are regarded as possible molecular candidates for receptor-operated cation channels. ${ }^{25}$ Taken together, these studies suggest that ciliary muscle function can be modulated by controlling the activation status of these different TRPC subfamily members. Increasing ciliary muscle contractility could cause trabecular meshwork relaxation and, in turn, enhance aqueous humour outflow. This outcome may be beneficial in treating hypertensive glaucoma resulting from a decline in conventional outflow facility.

\section{Lens}

Maintenance of intracellular $\mathrm{Ca}^{2+}$ levels is imperative for preserving lens clarity. ${ }^{26}$ This dependence is supported by findings indicating that lenses with cortical cataracts have intracellular $\mathrm{Ca}^{2+}$ levels that are above those in the physiological range. ${ }^{27}$ Accordingly, a better understanding of the mechanisms mediating control of intracellular $\mathrm{Ca}^{2+}$ levels is pertinent for identifying novel strategies to preserve lens transparency.

Calcium influx into lens epithelial cells is controlled by SOCE pathways whose stimulation of $\mathrm{Ca}^{2+}$ influx from the extracellular medium is higher in the equatorial than in the central anterior epithelial cells. This difference is attributable to the fact that the size of the ICS is larger in the equatorial than the central anterior epithelial cells. The larger store accounts for greater SOCE activity, since the activation of these cells is proportional to the magnitude of the decline in $\mathrm{Ca}^{2+}$ content subsequent to inhibition of ATP-dependent $\mathrm{Ca}^{2+}$ uptake into the ICS by thapsigargin. ${ }^{28}$ The involvement of either TRPV or TRPC isoforms as components of SOCE pathways remains unclear. Part of the reason for this uncertainty is that the pharmacological modulators available for characterising $\mathrm{Ca}^{2+}$ influx pathways lack adequate selectivity. For example, inhibitory sensitivity to lanthanum is used as a criterion for distinguishing between 
SOCE and TRPC involvement. At lower concentrations (ie in the nanomolar range), lanthanum inhibits SOCE, whereas higher concentrations are needed to block TRPC-containing channels. ${ }^{29,30}$ However, the cut-off between the different concentration ranges having these effects is poorly defined. Another complication is that, in the micromolar range, $\mathrm{Ca}^{2+}$ influx through TRPC4and TRPC5-containing pathways is potentiated. Nevertheless, the current understanding is that SOCE is the major pathway for $\mathrm{Ca}^{2+}$ influx in the lens.

\section{Retina}

Studies using Drosophila are relevant to understanding the functional importance of TRP channel expression in mediating phototransduction in mammals, since 13 of the 28 TRP proteins detected in mammals were found in Drosophila. ${ }^{31}$ Drosophila studies combine classical and modern genetic approaches to identifying genes and proteins that function in concert with TRPs to elicit phototransduction. As each Drosophila eye constitutes a reiteration of about 800 simple eyes, all the photoreceptor proteins are expressed in many cells. Their abundance greatly overcomes a problem inherent in many signalling systems - namely, the low abundance of the essential proteins and signalling molecules. ${ }^{32}$ Drosophila rhabdomeres are tightly packed microvilli, which contain photoreceptor cells. In these cells, light perception and phototransduction take place. Rhodopsin light excitation results in a light-induced current through activation of a GTP binding protein $(\mathrm{Gq})$-coupled PLC $\beta$.

Following cloning of the trp gene more than 20 years ago, TRP and its close homologue TRP-like (TRPL) became founding members of a large family of non-selective cation TRP channels, with a wide diversity in their modes of activation and in ion permeation properties. All of these channel types share a structural hallmark of six transmembrane domains (S1-S6) and a putative pore region between S5 and S6. Their C termini contain the S5-6 TRP motif, a calmodulin binding site, a proline-rich region and a PDZ motif. On the other hand, the amino motif contains a coiled-coil region and four ankyrin repeats. Four channel subunits assemble as homotetramers or heterotetramers to form a functional ion channel complex similar to $\mathrm{K}^{+}$channel architecture. ${ }^{33}$ The TRP channels in mammals that are most closely related to the Drosophila TRP channel are designated as TRP classical, or TRPC, channels. They comprise six subfamily genes $\operatorname{trpc} 1,3-7)$ encoding seven proteins (TRPC1-7), since $\operatorname{trpc} 2$ is a pseudogene. ${ }^{34}$

In Drosophila rhabdomeres, light absorption converts rhodopsin to active metarhodopsin, which activates Gq, resulting in PLC activation. PLC hydrolyses $\mathrm{PIP}_{2}$ to $\mathrm{IP}_{3}$ and diacylglycerol (DAG). DAG can be degraded to polyunsaturated fatty acids, releasing protons. Until very recently, the exact mechanism underlying the gating of the lightsensitive TRPC channels resulting from PLC activation was unclear. Huang and colleagues have helped to answer this question, by proposing that TRPC activation in Drosophila may be mediated by PLC-induced $\mathrm{PIP}_{2}$ depletion resulting in $\mathrm{IP}_{3}$ formation and proton release. ${ }^{35}$ In support of their model, the authors show that illumination in the microvilli leads to rapid acidification of approximately 0.2 units in less than 10 milliseconds, which sets up a proton gradient from the microvilli to the cell bodies. As this acidification in response to light does not occur in Drosophila lacking PLC, this finding is supportive of the notion that a decline in $\mathrm{pH}$ may elicit TRP activation. This dependence is further supported by the finding that characteristic TRP channel currents could also be activated by protonophore addition. ${ }^{35}$ They induce acidification that is independent of uncoupling mitochondrial aerobic metabolism, whereas other experiments have shown that sensitivity to protonophore-induced acidification is inversely related to the phosphoinositide levels in the microvillar plasma membrane. In addition, they show, in a heterologous expression system, that TRP channel activation is dependent on the size of the $\mathrm{pH}$ gradient imposed from the outside. ${ }^{35}$ Taken together, these studies offer a provocative new hypothesis for microvillar phototransduction and TRPC channel activation. Photoexcitation of TRP channel activity is a consequence of phosphoinositide depletion and 
acidification resulting from PLC-induced $\mathrm{PIP}_{2}$ hydrolysis and proton release associated with $\mathrm{IP}_{3}$ formation.

In the retina, microglial cells are needed for neuronal homeostasis and provide innate immune defence. Exposure of microglia to chronic stress is associated with various neurodegenerative diseases, including retinal dystrophies. One type of stress that can lead to the degeneration of retinal ganglion cells (RGCs) is elevated intraocular pressure (IOP). One procedure that simulates such a stress condition is exposing cultured microglia to an increase in IOP. After 48 hours' exposure to a pressure of $70 \mathrm{mmHg}$, the frequency of apoptosis increases. Pressure-induced TRPV1 activation contributes to this response, since apoptosis is diminished during exposure to a TRPV1 antagonist. Furthermore, such stress increases IL-6 release, which is also lessened by exposure to either a $\mathrm{Ca}^{2+}$ channel or a TRPV1 antagonist. Therefore, TRPV1 activation by pressure in microglia makes a substantial contribution to apoptosis through its mediation of intracellular $\mathrm{Ca}^{2+}$ transients. ${ }^{36}$ It was also shown that there is functional expression of mechanosensitive TRPV1 on RGCs. TRPV1 activation in RGCs by pressure contributes to increases in $\mathrm{Ca}^{2+}$ influx, as well as increases in TRPV1 expression. ${ }^{37}$ These pressure-induced effects suggest that plasma membrane deformation leads to TRPV1 activation, which results in RGC death. Taken together, TRPV1 expression in microglia and RGCs plays an important role in the maintenance of retinal homeostasis. The results also suggest that TRPV1 may be a novel drug target for reducing pressure-induced RGC death in glaucoma.

The retinal pigment epithelial (RPE) layer also plays essential roles in sustaining normal retinal function. It regulates the hydration and ionic composition of the subretinal space, as well as rod outer segment function. Another important function is to secrete cytokines that are essential for the maintenance of retinal health. It has been recently shown that this layer also expresses TRPV subfamily members whose functional activity is needed for the RPE layer to sustain retinal health. TRPV5 and TRPV6 expression was described, suggesting that these two most calcium-selective channels of the TRP superfamily contribute to the regulation of the subretinal space calcium composition accompanying light/dark transitions. ${ }^{38}$ In another study, TRPV2 was shown to control RPE release of vascular endothelial growth factor (VEGF). Insulin-like growth factor-1 (IGF-1) is a TRPV2 activator that selectively induces the intracellular $\mathrm{Ca}^{2+}$ transients during VEGF release. Control of this response is needed to reduce retinal neovascularisation, since wet age-related macular degeneration (AMD) is decreased or stabilised by treatment with anti-VEGF antibodies. These results suggest that reducing TRPV2 activation may provide another option for managing wet AMD. ${ }^{39}$

\section{Conclusions}

Activation of TRP channels in the eye elicits responses needed for ocular sensory and cellular functions. In mammals, TRP channel subunit proteins are encoded by 27 genes and are grouped into six different subfamilies, based on differences in amino acid sequence homology. Group 1 and Group 2 TRPs are only remotely related but share similar cation channel-forming structures of six transmembrane domains. Their cation selectivity and activation mechanisms are very diverse and depend on which TRP isomers combine with one another in either homomeric or heteromeric tetramer groupings. TRP channel activation induces a host of responses to variations in ambient temperature, pressure, osmolarity and $\mathrm{pH}$. In addition, their activation by injury induces inflammation, neovascularisation, pain and cell death, as well as wound healing. Although the signalling pathways mediating TRP control of these responses remain unclear, there is emerging interest in characterising their roles in inducing dry eye syndrome, glaucoma, cataracts and AMD pathophysiology. Such efforts could lead to the identification of novel drug targets for treating these diseases.

\section{Acknowledgment}

We thank Chad N. Brocker for his assistance in construction of the gene dendrogram. 


\section{References}

1. Minke, B., Wu, C. and Pak, W.L. (1975), 'Induction of photoreceptor voltage noise in the dark in Drosophila mutant', Nature Vol. 258, pp. $84-87$.

2. Harteneck, C., Plant, T.D. and Schultz, G. (2000), 'From worm to man: Three subfamilies of TRP channels', Trends Neurosci. Vol. 23, pp. $159-166$.

3. Montell, C. (2005), 'The TRP superfamily of cation channels', Sci. STKE Vol. 2005, p. re3.

4. Voets, T., Janssens, A., Droogmans, G. and Nilius, B. (2004), 'Outer pore architecture of a $\mathrm{Ca}^{2+}$-selective TRP channel', J. Biol. Chem. Vol. 279, pp. 15223-15230.

5. Venkatachalam, K. and Montell, C. (2007), 'TRP channels', Annu. Rev. Biochem. Vol. 76, pp. 387-417.

6. Clapham, D.E. (2003), 'TRP channels as cellular sensors', Nature Vol. 426 , pp. $517-524$

7. Vriens, J., Appendino, G. and Nilius, B. (2009), 'Pharmacology of vanilloid transient receptor potential cation channels', Mol. Pharmacol. Vol. 75, pp. $1262-1279$

8. Clapham, D.E., Montell, C., Schultz, G. and Julius, D. (2003), 'International Union of Pharmacology. XLIII. Compendium of voltagegated ion channels: Transient receptor potential channels', Pharmacol. Rev. Vol. 55, pp. 591-596.

9. Pedersen, S.F., Owsianik, G. and Nilius, B. (2005), 'TRP channels: An overview', Cell Calcium Vol. 38, pp. 233-252.

10. Birnbaumer, L., Yildirim, E. and Abramowitz, J. (2003), 'A comparison of the genes coding for canonical TRP channels and their $\mathrm{M}, \mathrm{V}$ and $\mathrm{P}$ relatives', Cell Calcium Vol. 33, pp. 419-432.

11. Murata, Y. and Masuko, S. (2006), 'Peripheral and central distribution of TRPV1, substance P and CGRP of rat corneal neurons', Brain Res. Vol. 1085, pp. 87-94.

12. Zhang, F, Yang, H., Wang, Z., Mergler, S. et al. (2007), 'Transient receptor potential vanilloid 1 activation induces inflammatory cytokine release in corneal epithelium through MAPK signaling', J. Cell. Physiol. Vol. 213, pp. 730-739.

13. Yamada, T., Ueda, T., Ugawa, S., Ishida, Y. et al. (2010), 'Functional expression of transient receptor potential vanilloid 3 (TRPV3) in corneal epithelial cells: Involvement in thermosensation and wound healing', Exp. Eye Res. Vol. 90, pp. 121-129.

14. Mergler, S., Garreis, F., Sahlmuller, M., Reinach, P.S. et al. (2010), 'Thermosensitive transient receptor potential channels (thermo-TRPs) in human corneal epithelial cells', J. Cell. Physiol., DOI: 10.1002/JCP.22514.

15. Pan, Z., Yang, H., Mergler, S., Liu, H. et al. (2008), 'Dependence of regulatory volume decrease on transient receptor potential vanilloid 4 (TRPV4) expression in human corneal epithelial cells', Cell Calcium Vol. 44, pp. 374-385.

16. Pan, Z., Wang, Z., Yang, H., Zhang, F. et al. (2011), 'TRPV1 activation is required for hypertonicity stimulated inflammatory cytokine release in human corneal epithelial cells', Invest. Ophthalmol. Vis. Sci. Vol. 52, No. 1, pp. 485-493.

17. Yang, H., Wang, Z., Capo-Aponte, J.E., Zhang, F. et al. (2010), 'Epidermal growth factor receptor transactivation by the cannabinoid receptor (CB1) and transient receptor potential vanilloid 1 (TRPV1) induces differential responses in corneal epithelial cells', Exp. Eye Res. Vol. 91, pp. 462-471

18. Yang, H., Mergler, S., Sun, X., Wang, Z. et al. (2005), 'TRPC4 knockdown suppresses epidermal growth factor-induced store-operated channel activation and growth in human corneal epithelial cells', J. Biol. Chem. Vol. 280, pp. 32230-32237.
19. Xie, Q., Zhang, Y., Cai Sun, X., Zhai, C. et al. (2005), 'Expression and functional evaluation of transient receptor potential channel 4 in bovine corneal endothelial cells', Exp. Eye Res. Vol. 81, pp. 5-14.

20. Mergler, S., Valtink, M., Coulson-Thomas, V.J., Lindemann, D. et al. (2010), 'TRPV channels mediate temperature-sensing in human corneal endothelial cells', Exp. Eye Res. Vol. 90, pp. 758-770.

21. Weinreb, R.N. and Khaw, P.T. (2004), 'Primary open-angle glaucoma', Lancet Vol. 363, pp. 1711-1720.

22. Abad, E., Lorente, G., Gavara, N., Morales, M. et al. (2008), 'Activation of store-operated $\mathrm{Ca}^{2+}$ channels in trabecular meshwork cells', Invest. Ophthalmol. Vis. Sci. Vol. 49, pp. 677-686.

23. Wiederholt, M., Thieme, H. and Stumpff, F. (2000), 'The regulation of trabecular meshwork and ciliary muscle contractility', Prog. Retin. Eye Res. Vol. 19, pp. 271-295.

24. Salmon, M.D. and Ahluwalia, J. (2010), 'Discrimination between receptor- and store-operated $\mathrm{Ca}(2+)$ influx in human neutrophils', Cell. Immunol. Vol. 265, pp. 1-5.

25. Sugawara, R., Takai, Y., Miyazu, M., Ohinata, H. et al. (2006), 'Agonist and antagonist sensitivity of non-selective cation channel currents evoked by muscarinic receptor stimulation in bovine ciliary muscle cells', Auton. Autacoid Pharmacol. Vol. 26, pp. 285-292.

26. Duncan, G., Williamsa, M.R. and Riacha, R.A. (1994), 'Calcium, cell signalling and cataract', Prog. Retin. Eye Res. Vol. 13, pp. 623-652.

27. Duncan, G. and Bushell, A.R. (1975), 'Ion analyses of human cataractous lenses', Exp. Eye Res. Vol. 20, pp. 223-230.

28. Rhodes, J.D., Russell, S.L., Illingworth, C.D., Duncan, G. et al. (2009), 'Regional differences in store-operated $\mathrm{Ca} 2+$ entry in the epithelium of the intact human lens', Invest. Ophthalmol. Vis. Sci. Vol. 50, pp. 4330-4336.

29. Gwack, Y., Srikanth, S., Feske, S., Cruz-Guilloty, F. et al. (2007), 'Biochemical and functional characterization of Orai proteins', J. Biol. Chem. Vol. 282, pp. 16232-16243.

30. Nilius, B., Owsianik, G., Voets, T. and Peters, J.A. (2007), 'Transient receptor potential cation channels in disease', Physiol. Rev. Vol. 87, pp. 165-217.

31. Gudermann, T. and Mederos y Schnitzler, M. (2010), 'Phototransduction: Keep an eye out for acid-labile TRPs', Curr. Biol. Vol. 20, pp. R149-R152.

32. Wang, T. and Montell, C. (2007), 'Phototransduction and retinal degeneration in Drosophila', Pflugers Arch. Vol. 454, pp. 821-847.

33. Gaudet, R. (2008). 'TRP channels entering the structural era', J. Physiol. Vol. 586, pp. 3565-3575.

34. Abramowitz, J. and Birnbaumer, L. (2009), 'Physiology and pathophysiology of canonical transient receptor potential channels', FASEB J Vol. 23, pp. 297-328.

35. Huang, J., Liu, C.H., Hughes, S.A., Postma, M. et al. (2010), 'Activation of TRP channels by protons and phosphoinositide depletion in Drosophila photoreceptors', Curr. Biol. Vol. 20, pp. 189-197.

36. Sappington, R.M. and Calkins, D.J. (2008), 'Contribution of TRPV1 to microglia-derived IL-6 and NFkappaB translocation with elevated hydrostatic pressure', Invest. Ophthalmol. Vis. Sci. Vol. 49, pp. 3004-3017.

37. Sappington, R.M., Sidorova, T., Long, D.J. and Calkins, D.J. (2009), 'TRPV1: Contribution to retinal ganglion cell apoptosis and increased intracellular $\mathrm{Ca} 2+$ with exposure to hydrostatic pressure', Invest. Ophthalmol. Vis. Sci. Vol. 50, pp. 717-728.

38. Kennedy, B.G., Torabi, A.J., Kurzawa, R., Echtenkamp, S.F. et al. (2010), 'Expression of transient receptor potential vanilloid channels TRPV5 and TRPV6 in retinal pigment epithelium', Mol. Vis. Vol. 16, pp. 665-675.

39. Cordeiro, S., Seyler, S., Stindl, J., Milenkovic, V.M. et al. (2010), 'Heatsensitive TRPV channels regulate VEGF-A secretion in retinal pigment epithelial cells', Invest. Ophthalmol. Vis. Sci. Vol. 51, pp. 6001-6008. 